\title{
Using operating room turnover time by anesthesia trainee level to assess improving systems-based practice milestones
}

\author{
Christopher Ryan Hoffman ${ }^{1 *} \mathbb{D}$, Michael Stuart Green ${ }^{1} \mathbb{D}$, Jasmine Liu', Usama lqbal ${ }^{1}$ and Kirtanaa Voralu ${ }^{2}$
}

\begin{abstract}
Background: Operating room (OR) metrics are frequently cited when optimizing cost efficacy and quality of care (Weiss et al, Characteristics of operating room procedures in U.S. hospitals, 2011: Statistical brief \#170, 2013; Macario A, Anesthesiology 105:237-240, 2006; Childers et al, JAMA Surg 153:e176233, 2018). Little has been reported to evaluate how anesthesia trainees change anesthesia-related efficiencies in the OR. Statistical correlation may demonstrate awareness and implementation of efficient systems-based practice.

Methods: Utilizing computerized OR information systems, specific data regarding anesthesia controlled turnover times were collected (546 data points) over the course of 4 months. The type of surgery performed, patient's American Society of Anesthesiologists (ASA) physical status and OR turnover times were compared for clinical anesthesia (CA) trainee levels CA1, CA2, CA3 and CRNAs. Standard descriptive statistics were computed. Analysis of variance (ANOVA) was performed to compare the average turnover time.

Results: Average OR turnover time was 31 min ranging from 8 to $60 \mathrm{~min}$. There was a significant difference between the OR turnover time of CA-1 (32 min) compared to CA-3 (29 min) $(p=0.017)$ and CA-1 compared to CRNA (30 min) ( $p=0.016)$. OR turnover time was significantly shorter in CA-3 and CRNA. The analysis showed no differences between OR turnover time of ASA categories.

Conclusions: These findings posit that trainees improve efficiency over time, but that education may for a time come at the expense of productivity. This trend may demonstrate a more profound understanding and mastery of a learner progressing in the graduate medical education system. This interplay plays a key role in clinical and academic shared success.
\end{abstract}

Keywords: Health resources, Resource allocation, Graduate medical education, Educational measurement, Anesthesia

\section{Background}

The need to optimize health care quality is substantial. Improvement is facilitated when providers regularly review performance, design interventions, and create teams to implement change. Operating rooms (ORs) are one of the most resource-consuming areas of a hospital to provide surgical care. A recent release from the US Healthcare Cost and Utilization Project found that the cost of a hospital stay after operative procedures was 2.5 times more expensive

\footnotetext{
* Correspondence: CRHoffman@gmail.com

${ }^{1}$ Department of Anesthesiology \& Perioperative Medicine, Drexel University College of Medicine, Hahnemann University Hospital, 245 N. 15th Street,

Suite 7502, MS 310, Philadelphia, PA 19102, USA

Full list of author information is available at the end of the article
}

than that of a hospitalized patient not requiring such procedures [1]. Optimal allocation of OR resources is a major priority to ensure cost-efficiency. Excess staffing, case start delays/cancelations, case duration, and case turnover are some contributors to resource allocation [2]. Time is the most precious resource of an OR. Cost attributable to fixed overhead to utilize an OR is an estimated $\$ 20$ per minute. When factoring in physician and nursing staff, hospitals charge between \$30-40 per minute depending on the complexity of the case [3]. Delayed case start times, prolonged turnover times, or equipment issues can hinder OR efficiency.

(C) The Author(s). 2018 Open Access This article is distributed under the terms of the Creative Commons Attribution 4.0 International License (http://creativecommons.org/licenses/by/4.0/), which permits unrestricted use, distribution, and reproduction in any medium, provided you give appropriate credit to the original author(s) and the source, provide a link to the Creative Commons license, and indicate if changes were made. The Creative Commons Public Domain Dedication waiver (http://creativecommons.org/publicdomain/zero/1.0/) applies to the data made available in this article, unless otherwise stated. 
Training anesthesia residents might increase process times, negatively influence OR efficiency, and increase waiting time for surgeons [4]. Teaching cases are associated with an increased anesthesia induction time by a mean of $4.5 \mathrm{~min}$ (standard deviation $3.2 \mathrm{~min}$ ) [5]. The introduction of new anesthesia residents causes a statistically significant effect on induction, emergence, and turnover times [6]. Other data suggests that decreases in anesthesia controlled time doesn't save enough time to increase scheduled surgical cases [7].

Although qualitative factors like professional satisfaction among surgeons and OR staff are a relevant priority, educational requisites are another aspect to consider [8]. One of the six core competencies dictated by The Accreditation Council for Graduate Medical Education (ACGME) is systems-based practice and "using system resources to facilitate cost-effective and safe non-subspecialty anesthesia care" [9]. Studies correlating training level and quality of care are scarce. Some show heterogeneous results with some inefficiencies found in specific procedure times or new exposure to subspecialty cases $[10,11]$. Efforts to improve cost efficiency in the academic setting may threaten thorough training in a fostering environment [12]. Ensuring efficiency for trainees is a challenging blend of hospital cost, ACGME core competency, and effective education. Previously, Eappen et al. concluded that OR metrics across three two-week periods in a single resident training year concluded "no clinically or economically meaningful adverse effect on the anesthesia-controlled time component of operating room efficiency" [6]. In this study, we hypothesized that turnover times are correlated with the level of training for anesthesiology residents. We aim to look at a broader amount of trainee levels over a larger span of time to observe for competency.

\section{Methods}

\section{Data collection}

Permission to retrospectively review OR case data was granted by the Institutional Review Board of our facility (Protocol \#1701005145). Turnover times were collected from patients 18-75 years old undergoing common ambulatory procedures under general anesthesia or monitored anesthesia care (MAC). Procedures that were delayed for external reasons (e.g. subsequent patient did not arrive, admission/post anesthesia care unit (PACU) delay, pending diagnostic test, surgeon or OR staff delay, etc.) were excluded. Turnover times were included only when staff remained the same between cases. Cases in which CA-1's were in the initial 8 weeks of orientation were excluded. Procedures with unique requirements that may skew results were excluded (e.g. emergencies, cases requiring preprocedure nerve blocks/vascular access/neuromonitors, cases transported directly to ICU). High-acuity or subspecialty cases unevenly assigned to upper-level residents were excluded to ensure each group of cases were similar. A sample size of 100 data points per group was determined. Past cases were reviewed one day at a time into the past until all the sample sizes were achieved, yielding a final data set of 546 data points.

\section{Turnover time}

Turnover time is defined as the time from the prior patient exiting the OR to the next patient entering the OR. We evaluated four groups: clinical anesthesia (CA) residents in the first, second, and third years, and certified registered nurse anesthetists (CRNA). We measured the OR turnover time in the CA-1, CA-2, and CA-3 resident levels and observed any difference in total or improved times among the residents or between residents and CRNAs.

Basic demographic data collected included the patient age, gender, and weight. Type and duration of surgery were also recorded. We did not attempt to control or modify the specific anesthetic techniques the groups used. Since it was a retrospective study, the nurse anesthetists and residents involved in the care of study patients were not aware of the study or of any specific parameters being measured. Thus, each anesthesiology trainee level developed and executed an anesthetic plan according to his/her usual practice.

\section{Statistical analysis}

Data analysis was performed with IBM SPSS (IBM Corp. Released 2016. IBM SPSS Statistics for Windows, Version 24.0. Armonk, NY: IBM Corp). Number of cases and percentages, mean, standard deviation (SD), median and range were obtained for OR turnover time according to ASA of previous OR case patient, ASA of next OR case patient and anesthesia trainee level. Analysis of variance (ANOVA) was performed to compare the average turnover time. Post-hoc analysis using Least Significant Difference (LSD) test were done to identify significant differences between the OR turnover time.

\section{Results}

A total of 546 data points were compared in this study. Data points were studied by CA-1 $(n=177)$, CA-2 $(n=$ $100)$, CA-3 $(n=99)$, and CRNA $(n=170)$ over 4 months. The overall average OR turnover time was 31 min ranging from 8 to $60 \mathrm{~min}$. Mean OR turnover time was $32 \mathrm{~min}$ for CA- 1 and CA-2 while $30 \mathrm{~min}$ for CRNAs and $29 \mathrm{~min}$ for CA-3. OR turnover time was significantly shorter in CA-3 and CRNA. The analysis showed no differences in OR turnover time among ASA categories (Tables 1 and 2).

A post-hoc analysis showed significant difference between the OR turnover time of CA-1 compared to CA-3 with significant $p$ value of 0.017 and CA-1 compared to CRNA with significant $\mathrm{p}$ value of 0.016 . 95\% confidence interval of mean difference was $0.54-5.44$ and $0.48-4.67$ respectively (Table 3 ). 
Table 1 Summary of OR turnover time according to ASA and anesthesia trainee level at Hahnemann University Hospital

\begin{tabular}{|c|c|c|c|}
\hline & \multirow{2}{*}{$\begin{array}{l}\text { Number of OR data points } \\
(n, \%)\end{array}$} & \multicolumn{2}{|c|}{ OR turnover time (minutes } \\
\hline & & $\begin{array}{l}\text { Mean } \\
\text { (SD) }\end{array}$ & $\begin{array}{l}\text { Median } \\
\text { (Range) }\end{array}$ \\
\hline Overall & $546(100)$ & $31(10)$ & $30(8-60)$ \\
\hline \multicolumn{4}{|c|}{ ASA of previous OR case patient } \\
\hline I & $35(6.4)$ & $30(11)$ & $27(14-56)$ \\
\hline$\|$ & $265(48.7)$ & $31(10)$ & $30(9-60)$ \\
\hline III & $242(44.5)$ & $30(10)$ & $29(8-57)$ \\
\hline IV & $2(0.4)$ & $28(11)$ & $28(20-35)$ \\
\hline \multicolumn{4}{|c|}{ ASA of next OR case patient } \\
\hline I & $27(5.0)$ & $29(8)$ & $27(19-49)$ \\
\hline$\|$ & $241(44.5)$ & $31(10)$ & $29(10-60)$ \\
\hline III & $272(50.3)$ & $31(10)$ & $30(8-57)$ \\
\hline IV & $1(0.2)$ & 24 & 24 \\
\hline \multicolumn{4}{|c|}{ Anesthesia trainee level } \\
\hline CA-1 & $177(32.4)$ & $32(10)$ & $31(10-58)$ \\
\hline $\mathrm{CA}-2$ & $100(18.3)$ & $32(10)$ & $30(9-60)$ \\
\hline CA-3 & $99(18.1)$ & $29(10)$ & $28(8-53)$ \\
\hline CRNA & $170(31.2)$ & $30(9)$ & $29(12-55)$ \\
\hline
\end{tabular}

\section{Discussion}

Our study shows that by the end of training there is a statistically significant reduction in turnover time. Even though this amounts to $2-3 \mathrm{~min}$, this is a $10 \%$ reduction in turnover time. A $10 \%$ improvement in any heavily valued performance metric warrants attention. This percentage may not have much opportunity for further improvement as general OR turnover is the same across most surgical cases. With multiple cases throughout the day this can accumulate to a sizeable amount of time. It may or may not be enough to fit additional OR cases but can ensure that OR staffing and scheduled time remains properly utilized and avoids overtime expense $[13,14]$. It is important for residents to appreciate achieving acceptable turnover times in order to perform competitively in future practices as well as establishing good rapport with staff and surgeons.

Prolonged turnover time can occur due to factors that are beyond our control. Our study tried to exclude external factors such as cases involving extra preparation, extremely sick patients, more complicated or prolonged surgeries, and delays due to non-anesthesiology team members. We removed the possibility of working faster under observation as our study is retrospective, so subjects were not aware times were being analyzed. Turnover time is when a patient exits the OR and the next patient enters the same OR [15]. These are recorded times which may not be properly recorded and don't denote an exact timeline. Times could be rounded up or down. We did attempt to eliminate turnover data points if there were delays admitting patients into preoperative holding or the PACU. Variable time it takes to transition care to PACU nursing, including PACU nurse unavailability and lengthy paperwork completion, can affect how long it takes for a provider to return to the OR and prepare for subsequent cases. A better measure may be when a provider is done signing out their patient in PACU to when their next patient is taken to the same OR. A potential bias we were unable to avoid is that the study was done only at our program, where many of our patients have multiple comorbidities. It is possible that

Table 2 Comparison OR turnover time according to ASA and anesthesia trainee level at Hahnemann University Hospital

\begin{tabular}{|c|c|c|c|c|c|}
\hline OR turnover time (minutes) & $\mathrm{N}$ & Mean (SD) & 95\% Confidence Interval of mean & F-test (df) & $p$-value \\
\hline \multicolumn{6}{|c|}{ ASA of previous OR case patient } \\
\hline । & 35 & $30(11)$ & 26,33 & $0.8(3,540)$ & 0.503 \\
\hline$\|$ & 365 & $31(10)$ & 30,33 & & \\
\hline III & 242 & $30(10)$ & 29,32 & & \\
\hline IV & 2 & $28(11)$ & $-68,123$ & & \\
\hline \multicolumn{6}{|l|}{ ASA of next OR case patient } \\
\hline । & 27 & $29(8)$ & 26,32 & $0.6(3,537)$ & 0.590 \\
\hline$\|$ & 241 & $30(10)$ & 29,32 & & \\
\hline III & 272 & $31(10)$ & 30,32 & & \\
\hline IV & 1 & 24 & - & & \\
\hline \multicolumn{6}{|l|}{ Anesthesia trainee level } \\
\hline CA-1 & 177 & $32(10)$ & 31,34 & $3.0(3,542)$ & 0.030 \\
\hline CA-2 & 100 & $32(10)$ & 30,34 & & \\
\hline CA-3 & 99 & $29(10)$ & 27,31 & & \\
\hline CRNA & 170 & 30 (9) & 28,31 & & \\
\hline
\end{tabular}


Table 3 Post-hoc analysis between OR turnover time and anesthesia trainee level Hahnemann University Hospital

\begin{tabular}{llll}
\hline OR turnover time (minutes) & Mean Difference (Standard Error) & $95 \%$ Confidence Interval of Mean Difference & $p$-value \\
\hline Anesthesia trainee level & & & \\
CA-1 vs CA-3 & $2.99(1.25)$ & $0.54,5.44$ & 0.017 \\
CA-1 vs CRNA & $2.57(1.07)$ & $0.48,4.67$ & 0.016 \\
\hline
\end{tabular}

we could have seen statistically significant difference between ASA classes if patients had fewer or different comorbidities. Excluding specialty cases in an attempt to control data may also skew results. Nursing staffing may be increased and attending anesthesiologist staffing may be one-on-one in this scenario, potentially streamlining turnover efforts. Retrospectively reviewing high-dimensional data may present a challenge when utilizing standard computational and statistical techniques. A larger scale prospective review of many anesthesia providers caring for patients with different ranges in comorbidities may elicit different results.

The anesthesia provider is one facet of a multidimensional process, and other sources of inefficiency contribute to delays. Complicated or delayed OR cleaning and subsequent surgical/ anesthesia equipment setup is not a reflection of the anesthesia provider but may be credited to their turnover time. Decreased turnover time may not be a reflection of the provider's capability. Medical student, student nurse anesthetist, or anesthesia assistant contributions toward case turnover is unaccounted for with unpredictable impact. Supervising anesthesiologists may affect turnover time depending on incoming patient evaluation, consent, and intravenous cannulation while the previous case is still in progress. Other undocumented factors could affect times and yet would not be shown in the record.

Turnover time is regarded as a critical component in optimizing OR management. Improvement has been correlated with better utilization of resources, reduction in staffing costs and wasted resources, and patient/staff satisfaction. Much emphasis has been paid upon anesthesiology-related metrics and how anesthesia trainees affect them. Prior reporting demonstrates mixed results overall with no significant increase in time for turnover. Our study is novel in its stratification amongst different class years and how turnover time may be improved with every successive year. The goal in identifying an improving trend theoretically translates to resident improvement in future practices. Optimal OR suites are able to turnover cases in $25 \mathrm{~min}$ or less [2]. Our study shows that residents can make significant enough progress to reach such a goal, and that senior residents are not a definite hindrance to OR efficiency. This is consistent with prior literature reporting anesthesia emergence times improving with ongoing training. Reviewing other metrics across residency training years may yield similar results.

The ACGME views "facilitating cost-effective and safe anesthesia care" as part of the systems-based practice milestone to evaluate a resident competency. There is little objective consensus on how to evaluate this. The study's intention of evaluating how resident training may affect cost efficacy in academic facilities also introduces the possibility of using OR metrics as a way to monitor progression. Given the amount of potential bias, this may not seem a practical nor an accurate reflection of the resident's progress. OR metrics more reliant on the provider that improve with experience (e.g. induction or emergence times, auditing drugs and resources utilization) may be better indicators. The "business of anesthesia" involves a managerial skill-set apart from clinical aptitude and may require qualitative monitoring [16]. Despite hospital emphasis on efficient turnover and increasing profit, the goal remains the same: efficiency without sacrificing education or patient safety. With these results, we hope to conduct further studies to stratify how improvements can be observed in resident training. Using metrics that anesthesia residents may have little control over may inappropriately assess competency and incorrectly monitor cost efficacy implications.

\section{Conclusions}

There was a significant difference between the OR turnover time of CA-1 compared to CA-3 $(p=0.017)$ and CA-1 compared to CRNA $(p=0.016)$. OR turnover time was significantly shorter in CA-3 and CRNA. The analysis showed no differences between OR turnover time of ASA categories. There is a statistically significant improvement and reduction in turnover time. Even though this amounts to just $2-3 \mathrm{~min}$, this amounts to a $10 \%$ reduction in turnover time. These findings demonstrate that anesthesia residents become more efficient over time. The relative inefficiencies of a resident's workflow at the beginning of training must be balanced by the education mission. These two points potentially have a substantial impact on optimizing education quality and cost efficacy in the healthcare system. Furthermore, what is not known is if this trend is simply a learning curve improved by experience, or a more profound understanding and mastery of a learner progressing in the graduate medical education system. How these competing priorities intertwine is pivotal to the discussions about the educational missions for health care institutions.

\footnotetext{
Abbreviations

ACGME: Accreditation Council for Graduate Medical Education;

ANOVA: Analysis of Variance; ASA: American Society of Anesthesiologists;

CA: Clinical Anesthesia; CRNA: Certified Nurse Anesthetist; df: Degrees of

Freedom; LSD: Least Significant Difference; MAC: Monitored Anesthesia Care;

OR: Operating Room; PACU: Post Anesthesia Care Unit; SD: Standard

Deviation
} 


\section{Acknowledgements}

Not applicable.

\section{Funding}

No funding to disclose.

\section{Availability of data and materials}

The datasets used and/or analysed during the current study are available from the corresponding author on reasonable request.

\section{Authors' contributions}

$\mathrm{CRH}$ is the corresponding author, aiding in data acquisition and primary manuscript writing. MSG is the principal investigator and edited the final manuscript. JL is a contributing author. $\mathrm{UI}$ acquired data and is a contributing author. KV developed and subsequently implemented the statistical plan/analysis. All authors read and approved the final manuscript.

\section{Ethics approval and consent to participate}

The Drexel University Institution Review Board (IRB) approved this retrospective review, Protocol \#1701005145. There was no patient consent for a retrospective review not including personal identifying information.

\section{Consent for publication}

Not applicable. No individual person's data was collected.

\section{Competing interests}

The authors declare that they have no competing interests.

\section{Publisher's Note}

Springer Nature remains neutral with regard to jurisdictional claims in published maps and institutional affiliations.

\section{Author details}

${ }^{1}$ Department of Anesthesiology \& Perioperative Medicine, Drexel University College of Medicine, Hahnemann University Hospital, 245 N. 15th Street, Suite 7502, MS 310, Philadelphia, PA 19102, USA. ${ }^{2}$ Houston Methodist Hospital, Houston, TX, USA.

Received: 31 May 2018 Accepted: 26 November 2018

Published online: 05 December 2018

\section{References}

1. Weiss AJ, Elixhauser A, Andrews RM. Characteristics of operating room procedures in U.S. hospitals, 2011: Statistical brief \#170. Rockville: Healthc Cost Util Proj Stat Briefs Rockv Agency Heal Care Policy Res; 2013.

2. Macario A. Are your hospital operating rooms "efficient"? A scoring system with eight performance indicators. Anesthesiology. 2006;105(2):237-40.

3. Childers $C P$, Maggard-Gibbons M. Understanding costs of care in the operating room. JAMA Surg. 2018;153(4):e176233.

4. Dexter F, Wachtel RE. Economic, educational, and policy perspectives on the preincision operating room period. Anesth Analg. 2006;103(4):919-21.

5. Davis EA, Escobar A, Ehrenwerth J, et al. Resident teaching versus the operating room schedule: an independent observer-based study of 1558 cases. Anesth Analg. 2006;103(4):932-7.

6. Eappen S, Flanagan $\mathrm{H}$, Bhattacharyya N. Introduction of anesthesia resident trainees to the operating room does not lead to changes in anesthesiacontrolled times for efficiency measures. Anesthesiology. 2004;101(5):1210-4.

7. Dexter F, Coffin S, Tinker JH. Decreases in anesthesia-controlled time cannot permit one additional surgical operation to be reliably scheduled during the workday. Anesth Analg. 1995;81(6):1263-8.

8. Vitez TS, Macario A. Setting performance standards for an anaesthesia department. J Clin Anaesth. 1998;10(2):166-75.

9. Culley DEA. The anesthesiology milestone project. J Grad Med Educ. 2014; 6(1 Suppl 1):15-28.

10. Posner KL, Freund PR. Resident training level and quality of anesthesia care in a university hospital. Anesth Analg. 2004;98(2):437-42.

11. Schuster M, Kotjan T, Fiege M, Goetz AE. Influence of resident training on anaesthesia induction times. Br J Anaesth. 2008;101(5):640-7.

12. Davis EA, Barash P. Teaching and operating room efficiency. Anesth Analg. 2007;104(4):992-3.
13. Dexter F, Jarvie C, Epstein RH. Lack of generalizability of observational studies' findings for turnover time reduction and growth in surgery based on the state of lowa, where from one year to the next, most growth was attributable to surgeons performing only a few cases per week. J Clin Anaesth. 2018;44:107-13.

14. Mclntosh C, Dexter F, Epstein $\mathrm{RH}$. The impact of service-specific staffing, case scheduling, turnovers, and first-case starts on anesthesia group and operating room productivity: a tutorial using data from an Australian hospital. Anesth Analg. 2006;103(6):1499-516.

15. Donham RT. Defining measurable OR-PR scheduling, efficiency, and utilization data elements: the Association of Anesthesia Clinical Directors procedural times glossary. Int Anesthesiol Clin. 1998 Winter;36(1):15-29.

16. Wasilko SM, Giordano C. Operating room management and the business of anesthesia: innovative solutions to bridge the educational gap in residency training programs. A A Pract. 2017;8(9):246.

\section{Ready to submit your research? Choose BMC and benefit from:}

- fast, convenient online submission

- thorough peer review by experienced researchers in your field

- rapid publication on acceptance

- support for research data, including large and complex data types

- gold Open Access which fosters wider collaboration and increased citations

- maximum visibility for your research: over $100 \mathrm{M}$ website views per year

At $\mathrm{BMC}$, research is always in progress.

Learn more biomedcentral.com/submissions 